\title{
Prognostic role of pre-sarcopenia and body composition with long-term outcomes in obstructive colorectal cancer: a retrospective cohort study
}

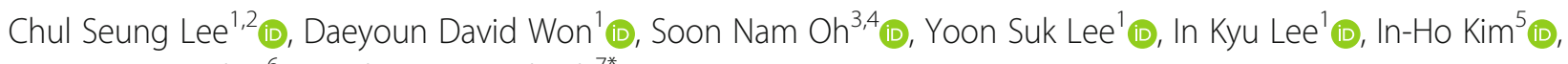
Moon Hyung Choi ${ }^{6}$ iD and Seong Taek $\mathrm{Oh}^{7^{*}}$ (D)

\begin{abstract}
Background: The clinical significance of pre-sarcopenia in colorectal cancer obstruction has not yet been described. The present study aimed to determine the short- and long-term oncologic impacts of pre-sarcopenia in obstructive colorectal cancer.

Methods: We retrospectively analyzed 214 patients with obstructive colon cancer between January 2004 and December 2013. Initial staging computed tomography (CT) scans identified pre-sarcopenia and visceral obesity by measuring the muscle and visceral fat areas at the third lumbar vertebra level. Both short-term postoperative and long-term oncologic outcomes were analyzed.

Results: Among all 214 patients, 71 (33.2\%) were diagnosed with pre-sarcopenia. Pre-sarcopenia had a negative oncologic impact in both disease-free survival (DFS) and overall survival (OS), (hazard ratio [HR] $=1.86,95 \%$ confidence interval [CI] 1.04-3.13, $p=0.037$, and $H R=1.92, \mathrm{Cl} 1.02-3.60, p=0.043$, respectively). Visceral adiposity, body mass index (BMI), and neutrophil-lymphocyte ratio (NLR) did not significantly impact DFS and OS.

Conclusion: Pre-sarcopenia is a clinical factor significantly associated with OS and DFS but not with short-term complications in obstructive colorectal cancer. In future, prospective studies should incorporate body composition data in patient risk assessments and oncologic prediction tools.
\end{abstract}

Keywords: Sarcopenia, Body composition, Colorectal neoplasms, Self-expandable metallic stents

\section{Background}

Sarcopenia is defined as a decrease in skeletal muscle volume and function [1] that has been reported to reflect patients' frailty. Major clinical guidelines have incorporated sarcopenia as a tool to assess cachexia in cancer patients [2]. Sarcopenia patients may possess unfavorable

\footnotetext{
* Correspondence: stoh@catholic.ac.kr

${ }^{7}$ Department of Surgery, Uijeongbu St. Mary's Hospital, College of Medicine, The Catholic University of Korea, 271, Cheonbo-Ro, Uijeongbu-si, Gyeonggi-do, 11765 Seoul, Republic of Korea

Full list of author information is available at the end of the article
}

nutritional and immunological factors [3] and show lower compliance to consecutive anti-tumor treatments such as radiotherapy, surgery, and chemotherapy [4].

Sarcopenia, especially in obese patients, is not easily characterized by overall weight loss or decreased body mass index (BMI) alone [5]. With an increased prevalence of obesity, patients with both sarcopenia and obesity (sarcopenic obesity) are at a higher risk of adverse outcomes [6] and mortality [7]. Thus, measuring the skeletal muscle mass and quality with BMI alone may not be appropriate in such cases. As reported,

(c) The Author(s). 2020 Open Access This article is licensed under a Creative Commons Attribution 4.0 International License, which permits use, sharing, adaptation, distribution and reproduction in any medium or format, as long as you give appropriate credit to the original author(s) and the source, provide a link to the Creative Commons licence, and indicate if changes were made. The images or other third party material in this article are included in the article's Creative Commons licence, unless indicated otherwise in a credit line to the material. If material is not included in the article's Creative Commons licence and your intended use is not permitted by statutory regulation or exceeds the permitted use, you will need to obtain permission directly from the copyright holder. To view a copy of this licence, visit http://creativecommons.org/licenses/by/4.0/ The Creative Commons Public Domain Dedication waiver (http://creativecommons.org/publicdomain/zero/1.0/) applies to the data made available in this article, unless otherwise stated in a credit line to the data. 
not only sarcopenia, but visceral obesity has also shown increased incidence of post-operative complication and delayed recovery [8, 9]. Further, preoperative computerized tomography (CT) scan by assessing the muscle area in the third lumbar vertebra (L3) region [10] appears to be one of the most widely researched topics in retrospective studies to measure skeletal muscle index (SMI) and to define pre-sarcopenia. Pre-sarcopenia is characterized by low muscle mass without impact on muscle strength [11].

Pre-sarcopenia is a significant prognostic factor in colorectal cancer patients. In colorectal cancer surgery, pre-sarcopenia has been reported to predict poorer postoperative short-term and oncologic long-term outcomes $[12,13]$. Pre-sarcopenia patients undergoing resection of colorectal liver metastases have a shorter median survival and lower disease-free survival (DFS) rates than non-pre-sarcopenia patients [14]. Additionally, presarcopenia was negatively associated with overall survival (OS) in locally advanced rectal cancer patients who underwent neoadjuvant chemoradiation therapy and curative resection [15]. Despite this, the clinical importance of pre-sarcopenia in obstructive colorectal cancer has not been fully described.

Obstructive colon cancer is a significant clinical event that affects the short- and long-term prognosis of patients. The rates of mortality and complications associated with emergency surgery for obstructive colorectal cancer are higher than those associated with elective surgeries $[16,17]$. Regarding long-term oncologic prognosis, colon cancer obstruction has a negative impact on DFS and OS $[18,19]$. Thus, treatment guidelines recommend adjuvant chemotherapy for this high-risk group [20].

Intestinal decompression using a self-expandable metallic colonic stent (SEMS) allows bowel preparation, medical stabilization, and optimization of comorbid illnesses, theoretically improving the patient's nutritional and inflammatory status. The Controlling Nutritional Status (CONUT) Score-a cumulative score calculated from the serum albumin level, total cholesterol level, and total lymphocyte count-is used as a nutritional screening tool. This score is easily calculated and found to be significantly associated with the Subjective Global Assessment (SGA) and Full Nutritional Assessment (FNA) tests [21]. Recent studies show that the preoperative CONUT score is an independent prognostic factor for cancer-specific survival and disease-free survival (DFS) in obstructive colorectal cancer patients [22].

Neutrophils play roles in both inflammation and cancer and have been implicated as key drivers of oncogenesis associated with postoperative inflammation [23, 24]. The neutrophil-to-lymphocyte ratio (NLR) has, hence, been proposed as an indicator of postsurgical inflammation-mediated tumor stimulation. NLR has also been reported to be an independent prognostic factor after resection of colorectal cancer [25-27].

Few studies have described the prognostic role of presarcopenia diagnosed by L3SMI in obstructive colorectal cancer, as compared to nutritional or inflammatory factors. The present study aims to determine if the short-term and long-term oncological effects of presarcopenia, diagnosed by L3SMI, may play a decisive role when compared to other factors in obstructive colorectal cancer.

\section{Materials and methods Definitions}

We have defined obstructive colorectal cancer as pathological confirmation of adenocarcinoma originated from the cecum to rectum with clinical symptom of obstruction (abdominal distention, pain, tenderness, and no stool passage) and radiological finding of obstruction in computed tomography (CT) scan or failure of scope passing beyond cancer lesion. Also, we included only complete obstruction to this definition, which clinically required immediate procedure. The surgeon finally decided whether patients had a complete obstruction or not. OS was defined as the period of time between the surgery and death of any cause. DFS was defined as the period of time between the surgery and disease of any cause. OS was calculated from date of surgery until death of any cause or censored at last follow-up.

\section{Subjects}

Between January 2004 and December 2013, a total of 214 consecutive patients with obstructive colorectal cancer were enrolled. The inclusion criteria were as follows: (1) Clinically (abdominal distention, pain, tenderness, and no stool passage) and radiologically confirmed malignant large bowel obstruction and (2) the patients who required immediate procedure due to complete obstruction. Colon perforation, metastatic cancer, and nonradical resection have been reported to be poor prognostic factors [28-30]. The exclusion criteria were as follows: (1) palliative bypass surgery, (2) palliative stoma surgery, (3) palliative bypass surgery, (4) metastatic cancer, and (5) colon perforation. During initial staging computed tomography (CT), body composition evaluations were performed at the level of the transverse process of third lumbar vertebra as previously described [15]. The total body fat area, visceral fat area, subcutaneous fat area, and abdominal circumference were measured automatically (TeraRecon Aquarius Workstation, TeraRecon, Foster City, California, USA), and visceral fat area was used to identify visceral adiposity. The skeletal muscle areas (psoas, para-spinal, transverse abdominis, rectus abdominis, internal oblique, and external oblique muscles) were measured using a commercial system 
(Advantage Windows workstation, GE Healthcare, Milwaukee, Wisconsin, USA) entailing Hounsfield unit thresholds between -29 to +150 [31]. To normalize the skeletal muscle area for patient height, skeletal muscle index (SMI) was calculated as the skeletal area $\left(\mathrm{cm}^{2}\right)$ divided by the square of the height $\left(\mathrm{m}^{2}\right)$. Pre-sarcopenia was defined using sex-specific cutoff values for SMI $\left(46.4 \mathrm{~cm}^{2} / \mathrm{m}^{2}\right.$ for men and $37.5 \mathrm{~cm}^{2} / \mathrm{m}^{2}$ for women) [32]. The cutoff CT visceral fat area for the classification of visceral obesity has not yet been standardized. Previous studies have used $100 \mathrm{~cm}^{2}$ and $130 \mathrm{~cm}^{2}$ as cutoffs [33]. The present study defined visceral obesity as an area of visceral fat of $130 \mathrm{~cm}^{2}$ and above. Left colon cancer was defined as cancer arising between the distal third of the transverse colon and the rectosigmoid junction of the colon. The CONUT score was calculated using the peripheral lymphocyte count, serum albumin, and total cholesterol concentrations. (1) Albumin concentrations of $\geq 3.5,3.0-3.49,2.5-2.99$, and $<2.5 \mathrm{~g} / \mathrm{dL}$ were scored as $0,2,4$, and 6 points, respectively; (2) total lymphocyte counts of $\geq 1600,1200-1599,800-1199$, and < 800/ $\mathrm{mm}^{3}$ were scored as $0,1,2$, and 3 points, respectively; and (3) total cholesterol concentrations of $\geq 180,140$ $179,100-139$, and $<100 \mathrm{mg} / \mathrm{dL}$ were scored as $0,1,2$, and 3 points, respectively. Accordingly, the sum of (1), (2), and (3) was the final CONUT score.

Emergency surgery or self-expandable metallic stent (SEMS) placement was performed based on previously published data [34]. The surgeon determined the operative method for each case considering the patient's medical status and tumor location. A SEMS (HANARO stent, M.I. Tech Co., Ltd, Seoul, South Korea, or Niti-S stent, Taewoong Medical, Co., Ltd, Gyeonggido, South Korea) was inserted through the working channel over the guidewire under fluoroscopic guidance.

This study was approved by the St. Mary's Hospital Research Ethics Board (KC19RESI0152), and informed consent was obtained from all participants.

\section{Statistical analysis}

Chi-square and Fisher's exact tests were performed for association analysis. $P$ values $<0.05$ were considered statistically significant. Differences between groups were evaluated using Student's $t$ and $x^{2}$ tests for continuous and categorical variables, respectively. Categorical variables were reported as numbers and percentages, and distribution of continuous characteristics was reported as median (interquartile range [IQR]) or mean \pm standard deviation (SD). OS and DFS curves were analyzed using the Kaplan-Meier method and compared by logrank test for univariate analysis. Hazard ratios (HRs) were estimated with a Cox proportional hazards regression model. A multivariable Cox regression analysis was carried out to examine whether the different variables were associated with disease recurrences and overall survival (OS). For backward conditional Cox proportional hazards analysis, variables were chosen according to $p<$ 0.05 in univariate analysis. Statistical analyses were performed using SPSS version 24.0 (IBM SPSS Statistics ${ }^{\circ}$, Armonk, NY, USA). All of the tests were 2 sided, and $p$ $<0.05$ was considered significant.

\section{Results}

A total of 214 patients (126 men and 90 women) were enrolled in this study. The detailed demographics and clinical treatment courses according to presarcopenia status are summarized in Table 1 . Seventy-one patients had pre-sarcopenia based on initial CT. Tumor-related factors (tumor location, carcinoembryonic antigen [CEA]), pathologic factors (stage, lymph node status, lymph-vascular invasion), patient-related factors (American Society of Anesthesiologist [ASA] physical status), and nutritional factor (CONUT score) did not differ significantly according to pre-sarcopenia status (Table 1). Pre-sarcopenia was observed more frequently in male patients than in female patients ( 51 men, $70.8 \%$ vs 75 men, $52.1 \%, p=0.008$ ). Table 2 shows the association between pre-sarcopenia and body composition factors. The mean BMIs in the non-pre-sarcopenia and presarcopenia groups did not show significant difference based on sex ( $p=0.486$ and 0.687 , respectively).

At a median follow-up of 54 months (range, 2-137), there was no significant difference of short-term outcomes between pre-sarcopenia and non- pre-sarcopenia groups. A comparison of the clinical outcomes between patients with or without pre-sarcopenia is summarized in Table 3. A comparison of the clinical outcomes between patients with or without pre-sarcopenia is summarized in Table 3. There were no significant differences between stent insertion and pre-sarcopenia in obstructive colon cancer patients or in the frequencies of complications such as anastomosis leak, postoperative ileus, and surgical site infection. Patients with presarcopenia showed a significantly shorter median DFS than patients without pre-sarcopenia (median [IQR] 29.5 [11.8-109.0] versus 42.0 [24.0-133.0]; $p=0.007$ ). Similarly, overall survival (OS) was shorter in patients with pre-sarcopenia than in patients without pre-sarcopenia (median [IQR] 38.0 [21.8-60.3] versus 58.0 [26.0-81.0] days; $p=0.047$ ) (Table 3).

During the follow-up period, 53 cancer recurrence events (24.5\%) were observed. Patients with presarcopenia showed a significantly shorter DFS than patients without pre-sarcopenia (hazard ratio $[\mathrm{HR}]=1.860$, 95\% confidence interval $[\mathrm{CI}] 1.08-3.22, p=0.023$ ) (Fig. 1a; Table 4). CONUT score, which represents nutritional state, were not associated with DFS $(p=0.892)$. Visceral 
Table 1 Clinicopathologic characteristics according to pre-sarcopenia status

\begin{tabular}{|c|c|c|c|}
\hline & Non-pre-sarcopenia $(N=143)$ & Pre-sarcopenia $(N=71)$ & $P$ \\
\hline Sex, male (\%) & $75(52.1)$ & $51(70.8)$ & 0.008 \\
\hline Age (years), > 70 (\%) & $29(20.1)$ & $21(29.2)$ & 0.138 \\
\hline Location (\%) & & & 0.958 \\
\hline Right & $32(22.2)$ & $16(22.2)$ & \\
\hline Left & $94(65.3)$ & $46(63.9)$ & \\
\hline Rectum & $18(12.5)$ & $10(13.9)$ & \\
\hline Preoperative CEA, $\geq 5$ (\%) & $55(40.4)$ & $26(40.0)$ & 0.952 \\
\hline Neutrophil lymphocyte ratio, $\geq 5$ (\%) & $54(76.1)$ & $17(23.9)$ & 0.932 \\
\hline ASA (\%) & & & 0.466 \\
\hline 1 & $42(29.2)$ & $18(25.0)$ & \\
\hline$\|$ & $91(63.2)$ & $46(63.9)$ & \\
\hline III & $11(7.6)$ & $7(9.7)$ & \\
\hline IV & $0(0.0)$ & $1(1.4)$ & \\
\hline Operation methods (\%) & & & 0.530 \\
\hline Laparoscopy & 57 (39.6) & $23(31.9)$ & \\
\hline Open & $75(52.1)$ & $43(59.7)$ & \\
\hline Conversion & $12(8.3)$ & $6(8.3)$ & \\
\hline Stage $(\%)$ & & & 0.092 \\
\hline$\|$ & $48(33.3)$ & $16(25.0)$ & \\
\hline III & $96(66.7)$ & $56(77.8)$ & \\
\hline CONUT score & $2.6 \pm 2.2$ & $3.2 \pm 2.3$ & 0.181 \\
\hline LN harvest & $27.4 \pm 14.0$ & $26.8 \pm 15.1$ & 0.781 \\
\hline Tumor size $(\mathrm{cm})$ & $5.5 \pm 2.1$ & $5.6 \pm 2.2$ & 0.791 \\
\hline Lymphatic invasion, Yes (\%) & $92(63.9)$ & $44(61.1)$ & 0.690 \\
\hline Vascular Invasion, Yes (\%) & $22(15.3)$ & $13(18.1)$ & 0.601 \\
\hline Perineural invasion, Yes (\%) & $52(36.1)$ & $28(38.9)$ & 0.690 \\
\hline Differentiation (\%) & & & 0.840 \\
\hline Well & $9(6.3)$ & $4(5.6)$ & \\
\hline Moderate & $125(86.8)$ & $63(87.5)$ & \\
\hline Poor & $10(6.9)$ & $5(6.9)$ & \\
\hline
\end{tabular}

CEA carcinoembryonic antigen, ASA American Society of Anesthesiologist physical status, LN lymph node, CONUT Controlling Nutritional Status

Table 2 Associations between body composition factors and pre-sarcopenia

\begin{tabular}{|c|c|c|c|c|c|c|}
\hline \multirow[b]{2}{*}{ Sex } & \multicolumn{3}{|c|}{ Non-pre-sarcopenia } & \multicolumn{3}{|l|}{ Pre-sarcopenia } \\
\hline & Male $(N=75)$ & Female $(N=69)$ & $P$ & Male $(N=51)$ & Female $(N=21)$ & $P$ \\
\hline BMI $\left(\mathrm{kg} / \mathrm{m}^{2}\right)$ & $23.9 \pm 2.6$ & $23.6 \pm 3.2$ & 0.486 & $21.2 \pm 2.7$ & $21.0 \pm 3.4$ & 0.687 \\
\hline Visceral fat area $\left(\mathrm{cm}^{2}\right)$ & $129.2 \pm 69.6$ & $87.2 \pm 56.8$ & $<0.001$ & $101.6 \pm 64.0$ & $52.7 \pm 39.8$ & $<0.001$ \\
\hline Subcutaneous fat area $\left(\mathrm{cm}^{2}\right)$ & $88.9 \pm 39.8$ & $136.9 \pm 57.9$ & $<0.001$ & $68.3 \pm 39.7$ & $87.2 \pm 45.3$ & 0.083 \\
\hline Abdominal circumference $(\mathrm{cm})$ & $86.9 \pm 8.8$ & $82.9 \pm 9.2$ & 0.008 & $83.4 \pm 9.3$ & $74.3 \pm 9.6$ & $<0.001$ \\
\hline $\mathrm{SMI}\left(\mathrm{cm}^{2} / \mathrm{m}^{2}\right)$ & $52.7 \pm 4.2$ & $43.8 \pm 4.4$ & $<0.001$ & $118.2 \pm 11.5$ & $83.1 \pm 7.0$ & $<0.001$ \\
\hline
\end{tabular}


Table 3 Post-operative and clinical outcomes according to pre-sarcopenia status

\begin{tabular}{|c|c|c|c|}
\hline & Non-pre-sarcopenia $(N=144)$ & Pre-sarcopenia $(N=72)$ & $P$ \\
\hline Postoperative hospital days (median [IQR]) & $13.0[10.0-17.0]$ & $14.0[10.8-17.0]$ & 0.450 \\
\hline Stent, yes (\%) & $80(55.6)$ & $37(51.4)$ & 0.562 \\
\hline Stoma, yes (\%) & $25(17.4)$ & $16(22.2)$ & 0.390 \\
\hline Emergency operation, yes (\%) & $9(6.3)$ & $6(8.3)$ & 0.570 \\
\hline Transfusion, yes (\%) & $66(45.8)$ & $41(56.9)$ & 0.124 \\
\hline Complications (\%) & & & 0.906 \\
\hline No & $119(82.6)$ & $58(80.6)$ & \\
\hline Minor $^{\mathrm{a}}$ & $17(11.8)$ & $9(12.5)$ & \\
\hline Major ${ }^{b}$ & $8(5.6)$ & $5(6.9)$ & \\
\hline Anastomosis leak (\%) & $7(4.9)$ & $4(5.6)$ & 0.827 \\
\hline Postoperative ileus (\%) & $8(5.6)$ & $3(4.2)$ & 0.662 \\
\hline Surgical site infection (\%) & $6(4.2)$ & $2(2.8)$ & 0.610 \\
\hline Anastomosis site bleeding (\%) & $2(1.4)$ & $0(0.0)$ & 0.315 \\
\hline Urinary retention (\%) & $2(1.4)$ & $2(2.8)$ & 0.475 \\
\hline Atelectasis (\%) & $4(2.8)$ & $3(4.2)$ & 0.587 \\
\hline Pleural effusion & $1(0.7)$ & $1(1.4)$ & 0.615 \\
\hline Asthma attack & $1(0.7)$ & $0(0.0)$ & 0.478 \\
\hline Recurrence, yes (\%) & $30(20.8)$ & $23(31.9)$ & 0.074 \\
\hline Death, yes (\%) & $20(13.9)$ & $21(29.2)$ & 0.007 \\
\hline Disease-free survival time (months) (median [IQR]) & $42.0[24.0-133.0]$ & $29.5[11.8-109.0]$ & 0.007 \\
\hline Overall-survival time (months) (median [IQR]) & $58.0[26.0-81.0]$ & $38.0[21.8-60.3]$ & 0.047 \\
\hline
\end{tabular}

${ }^{\mathrm{a}}$ Clavien-Dindo Classification < IIIb

${ }^{\mathrm{b}}$ Clavien-Dindo Classification $\geq \mathrm{Illb}$

adiposity and BMI were not associated with DFS ( $p=$ $0.206,0.265$, and 0.702, respectively) (Fig. 1b, c).

On univariate analyses, Age, ASA, stage, vascular invasion, CONUT score, and pre-sarcopenia were associated with OS. Pre-sarcopenia patients showed a significantly shorter $\mathrm{OS}$ than non-pre-sarcopenia patients $(\mathrm{HR}=$ 2.38, CI 1.29-4.41, $p=0.004$ ) (Fig. 2a). In multivariable adjusted analysis, pre-sarcopenia ( $\mathrm{HR}=1.92 \mathrm{CI} 1.02$ to $3.60, p=0.043)$ was retained as a risk factor of OS for a 5 -year OS (Table 5). Visceral adiposity and BMI were not associated with OS $(p=0.061,0.215$, and 0.527 , respectively; Fig. 2b, c).

\section{Discussion}

Pre-sarcopenia was negatively associated with a longterm oncological prognosis. Pre-sarcopenia patients showed significantly lower OS and DFS. However, there were no significant differences in short-term postoperative outcomes. Body composition factors other than presarcopenia did not significantly impact the patient's survival. Pre-sarcopenia was negatively associated with a long-term oncological prognosis. Pre-sarcopenia patients showed significantly lower OS and DFS. However, there were no significant differences in short-term postoperative outcomes. Body composition factors other than pre-

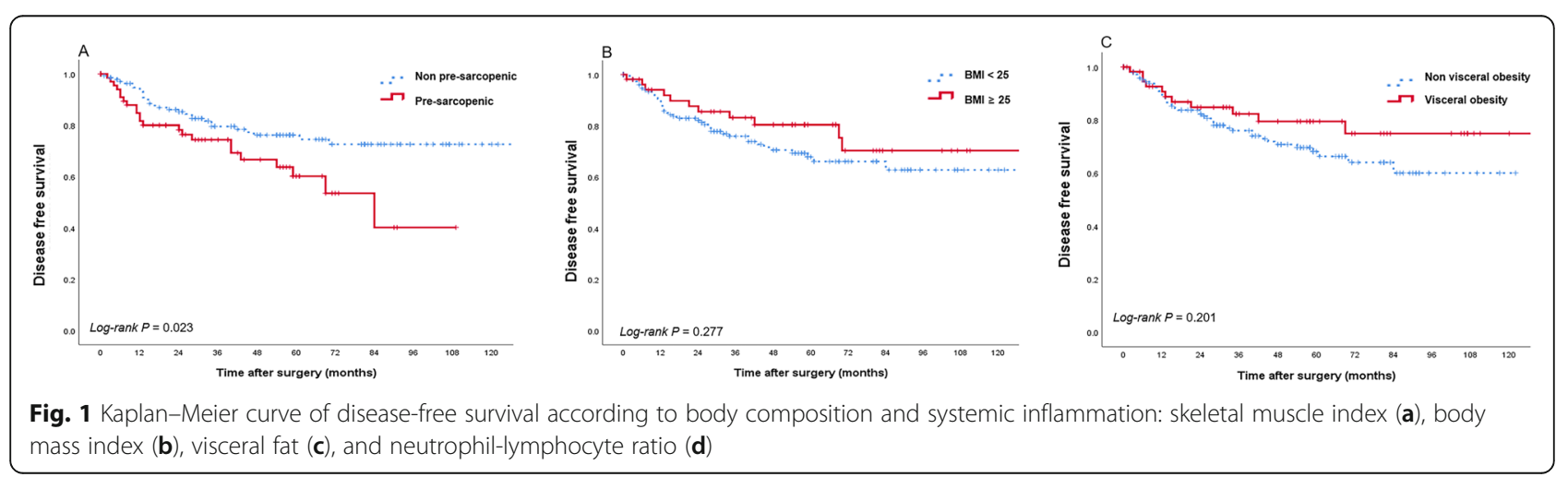


Table 4 Disease-free survival analysis by clinicopathologic variables and body composition factors

\begin{tabular}{|c|c|c|c|c|}
\hline \multirow[t]{3}{*}{ Variable } & \multicolumn{4}{|l|}{ Analysis } \\
\hline & \multicolumn{2}{|l|}{ Univariate } & \multicolumn{2}{|l|}{ Multivariable } \\
\hline & $\mathrm{HR}[95 \% \mathrm{Cl}]$ & $P$ value & $\mathrm{HR}[95 \% \mathrm{Cl}]$ & $P$ value \\
\hline Sex, male & $1.09[0.63-1.88]$ & 0.818 & - & - \\
\hline Age $>60$ & $1.03[0.60-1.78]$ & 0.908 & & \\
\hline ASA (I vs ||$+|||+| V)$ & $1.08[0.60-1.94]$ & 0.790 & - & - \\
\hline Stage (II vs III) & $6.27[2.13-17.39]$ & $<0.001$ & $3.64[1.14-11.67]$ & 0.030 \\
\hline Vascular invasion & $2.05[1.09-3.84]$ & 0.026 & $1.77[0.92-3.37]$ & 0.087 \\
\hline Lymphatic invasion & $3.81[1.72-8.44]$ & 0.001 & $1.76[0.70-4.43]$ & 0.229 \\
\hline Pre-operative CEA level $>5$ & $2.17[1.25-3.75]$ & 0.006 & $2.226[1.28-3.87]$ & 0.005 \\
\hline Neutrophil lymphocyte ratio $\geq 5$ & $0.31[0.35-1.34]$ & 0.702 & & \\
\hline Differentiation (well vs moderate +poor) & $1.70[0.41-7.01]$ & 0.461 & - & - \\
\hline $\mathrm{BMI} \geq 25$ & $0.69[0.36-1.35]$ & 0.265 & - & - \\
\hline Visceral obesity $\geq 130[33]$ & $0.65[0.34-1.27]$ & 0.206 & - & - \\
\hline Subcutaneous fat area $\left(\mathrm{cm}^{2}\right)>92^{\mathrm{a}}$ & $0.80[0.47-1.37]$ & 0.417 & - & - \\
\hline Abdominal circumference $(\mathrm{cm})>83^{\mathrm{a}}$ & $0.89[0.52-1.52]$ & 0.663 & - & - \\
\hline CONUT score $>$ 7[22] & $0.91[0.22-3.79]$ & 0.892 & - & - \\
\hline Pre-sarcopenia, men $<46.4 \mathrm{~cm}^{2}$, women $<37.5 \mathrm{~cm}^{2}$ [32] & $1.83[1.08-3.22]$ & 0.023 & $1.86[1.04-3.13]$ & 0.037 \\
\hline
\end{tabular}

CI confidence interval, ASA American Society of Anesthesiologist physical status, CEA carcinoembryonic antigen, BMI body mass index, CONUT Controlling Nutritional Status

${ }^{\mathrm{a} M e d i a n}$ value

sarcopenia did not significantly impact the patient's survival.

Pre-sarcopenia comprises both muscle loss and its dysfunction, which induce contractile impairment and metabolic and endocrine abnormalities. It affects wholebody metabolism and the immune or inflammatory response $[35,36]$. The loss of muscle and the accumulation of intramuscular fat might be associated with metabolic syndrome via a complex interplay of factors including oxidative stress, proinflammatory cytokines, insulin resistance, hormonal changes, and mitochondrial dysfunction [37]. These factors may have contributed to long-term survival.

The results of our study suggest that pre-sarcopenia is a negative prognostic factor for both OS and DFS in patients with obstructive colorectal cancer. To our knowledge, this is the first report to describe the prognostic impact of pre-sarcopenia specifically in patients with obstructive colorectal cancer. Other studies reported pre-sarcopenia to have a negative effect on OS in patients undergoing resection for locally advanced rectal cancer after neoadjuvant chemoradiation therapy [15] and as an independent predictor of worse OS and DFS in stage I to III colorectal cancer [38].

Early studies of pre-sarcopenia were often based on the work by Prado et al. [39]. In their study, they included pre-sarcopenia data based on solid tumors of the lung or gastrointestinal tract from patients referred to a regional medical oncology service in Canada. However, because there are significant differences in body composition between different ethnicities, more data are needed from the Asian populations [40]. Moreover,
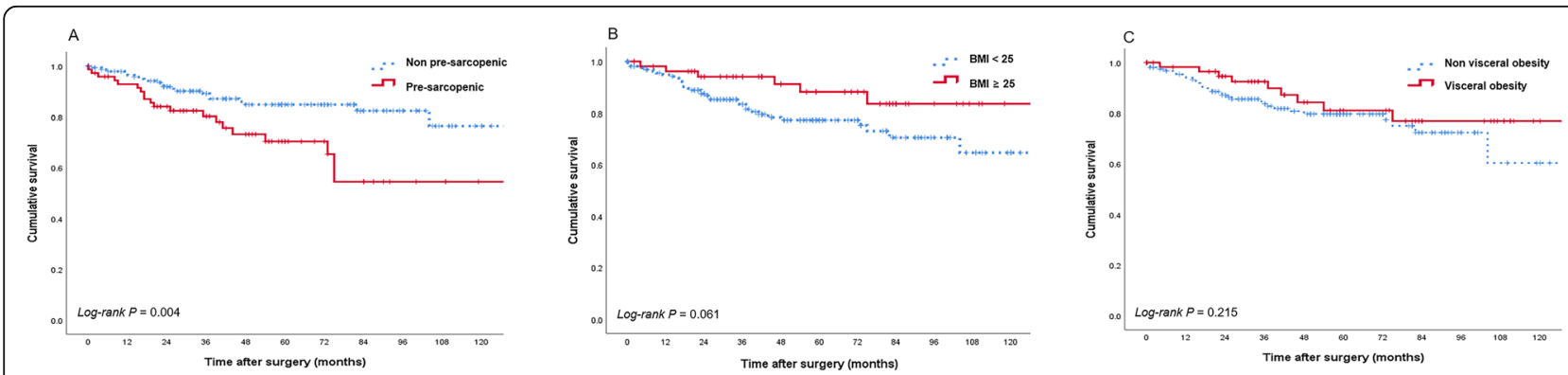

Fig. 2 Kaplan-Meier curve of overall survival according to body composition and systemic inflammation: skeletal muscle index (a), body mass index (b), visceral fat (c), neutrophil-lymphocyte ratio (d) 
Table 5 Overall survival analysis by clinicopathologic variables and body composition factors

\begin{tabular}{|c|c|c|c|c|}
\hline \multirow[t]{3}{*}{ Variable } & \multicolumn{4}{|l|}{ Analysis } \\
\hline & \multicolumn{2}{|l|}{ Univariate } & \multicolumn{2}{|l|}{ Multivariable } \\
\hline & $\mathrm{HR}[95 \% \mathrm{Cl}]$ & $P$ value & $\mathrm{HR}[95 \% \mathrm{Cl}]$ & Pvalue \\
\hline Sex, male & $1.43[0.75-2.74]$ & 0.284 & - & - \\
\hline Age $>60$ & $2.88[1.37-6.04]$ & 0.005 & $2.51[1.14-5.56]$ & 0.023 \\
\hline ASA (I vs ||$+|||+| V)$ & $2.27[1.01-5.14]$ & 0.048 & $1.99[0.84-4.71]$ & 0.118 \\
\hline Stage (II vs III) & $2.78[1.17-6.64]$ & 0.021 & $2.29[0.94-5.59]$ & 0.069 \\
\hline Vascular invasion & $2.14[1.04-4.40]$ & 0.039 & $1.90[1.01-3.57]$ & 0.030 \\
\hline Lymphatic invasion & $1.97[0.94-4.13]$ & 0.074 & - & - \\
\hline Pre-operative CEA level > 5 & $1.63[0.84-3.13]$ & 0.147 & - & - \\
\hline Neutrophil lymphocyte ratio $\geq 5$ & $0.78[0.36-1.70]$ & 0.529 & & \\
\hline Differentiation (well vs moderate +poor) & $0.66[0.21-1.67]$ & 0.322 & - & - \\
\hline $\mathrm{BMI} \geq 25$ & $0.45[0.19-1.06]$ & 0.069 & - & - \\
\hline Visceral obesity $\geq 130[33]$ & $0.63[0.22-1.32]$ & 0.220 & - & - \\
\hline Subcutaneous fat area $\left(\mathrm{cm}^{2}\right)>92^{\mathrm{a}}$ & $0.81[0.43-1.51]$ & 0.504 & - & - \\
\hline Abdominal circumference $(\mathrm{cm})>83^{\mathrm{a}}$ & $0.81[0.44-1.52]$ & 0.817 & - & - \\
\hline CONUT score $>$ 7[22] & $2.27[0.53-9.61]$ & 0.254 & & \\
\hline Pre-sarcopenia, men $<46.4 \mathrm{~cm}^{2}$, women $<37.5 \mathrm{~cm} 2[32]$ & 2.38 [1.29-4.41] & 0.004 & $1.92[1.01-3.57]$ & 0.047 \\
\hline
\end{tabular}

CI confidence interval, ASA American Society of Anesthesiologist physical status, BMI body mass index, CEA carcinoembryonic antigen, CONUT Controlling Nutritional Status

${ }^{\mathrm{a} M e d i a n}$ value

more data are needed to assess the optimal cutoff value for pre-sarcopenia for each ethnicity [13]. The Asian cut-off value for pre-sarcopenia should be different from the one used in Western countries.

Pre-sarcopenia was associated with a significantly increased risk of developing major complications [41]. However, one study reported that pre-sarcopenia was not a predictor of postoperative complications [8]. In the present study, no significant differences in minor $(11.8 \%$ vs. $12.5 \%)$ or major postoperative complications $(5.6 \%$ vs. $6.9 \%)$ were observed between non-pre-sarcopenia and pre-sarcopenia patients (Table 3). All surgeries were performed at the three tertiary-referral hospitals where more than 100 colorectal cancer patients are treated annually by seven independent surgeons. These surgeons were qualified through live demonstrations held by the Korean Laparoscopic Colorectal Surgery Study Group, and each submitted a videotape of their laparoscopic rectal surgery, which was subsequently reviewed by a trial steering committee to assess the surgeon's oncological technique [42].

Visceral obesity was reported to be a significant prognostic factor in predicting DFS in patients with resectable colorectal cancer [43]. Similarly, viscerally obese patients with rectal cancer have poorer DFS [44]. In contrast, BMI measurements were not correlated with any survival outcomes [43, 44]. Our data demonstrated that pre-sarcopenia was negative associated with visceral obesity (Table 2), but there were no other significant differences in predicting DFS and OS in patients with obstructive colorectal cancer. A lower BMI was correlated with pre-sarcopenia but not with prognosis. SMI was the most meaningful prognostic value among body composition factors.

According to a recent meta-analysis study [13], most studies $(11 / 22,50 \%)$ used SMI when applying muscle mass criteria in CT scan-based assessments of skeletal muscle index (SMI). Their defined cutoff values varied between 53.5 and $40.8 \mathrm{~cm}^{2} / \mathrm{m}^{2}$ for men and 46.4 and $34.9 \mathrm{~cm}^{2} / \mathrm{m}^{2}$ for women. To define a pre-sarcopenia cutoff value for this study, we analyzed the association between body composition factors and pre-sarcopenia in four different studies that included an Asian study population $\left(46.4,43.75,43.2\right.$, and $40.8 \mathrm{~cm}^{2} / \mathrm{m}^{2}$ for men and $37.5,41.10,35.3$, and $34.9 \mathrm{~cm}^{2} / \mathrm{m}^{2}$ for women for each study, respectively) [32, 45-47]. Among these, the cutoff value proposed by Takagi was applied differently to men and women and the inclusion criteria selected in their study was similar to ours. Additionally, their selected cutoff value did not differ significantly from the cutoff value of other Asian references. Thus, we used the same cutoff value proposed by Takagi [32], which was ideal for our study in Asian patients.

There are some limitations to this study. One possible limitation of the study is the definition of pre-sarcopenia. It is based on the definition by the European Working Group on Sarcopenia in Older People (EWGSOP) and the Asian Working Group for Sarcopenia (AWGS) that relies 
on the presence of low muscle mass and low muscle function (muscle strength and physical performance) [11]. However, there is still no uniform standard to measure and define sarcopenia, including the protocol of grip strength measures till date $[48,49]$. Pre-sarcopenia can be measured by CT scan by assessing the muscle area in the L3 region [10]. This method is commonly used both to measure SMI and to define pre-sarcopenia.

Another limitation of the study is bias of SMI results for the measurement of pre-sarcopenia. There were no association between pre-sarcopenia and nutrition status in this study (Supplementary Table 1\&2). That is, clinicians were not blinded to the SMI results. Since this is a retrospective study, surgeons may have been more careful to perioperative management such as nutrition support to those with pre-sarcopenia during and after surgery. It is apparent that patients who had pre-sarcopenia were well managed in this study, as reflected by the complication rate with no significant difference shown between pre-sarcopenia and non-pre-sarcopenia patients. Owing to these limitations, the conclusion of our study may not be definite, and hence, the differences in postoperative outcome between pre-sarcopenia and long-term outcomes in obstructive colon cancer must be probed further with prospective randomized studies. Despite these limitations, the results of our study emphasized that the pre-sarcopenia diagnosed by L3SMI showed oncologic significance in obstructive colorectal cancer.

Treatment of obstructive colorectal cancer may occasionally require colonic stent implantation to improve the patient's condition along with adjuvant chemotherapy that is often administered to these patients. Patients with obstructive colon cancer generally have poor prognosis and suffer large clinical burdens, including pre-sarcopenia.

The CONUT score was originally developed as a tool for nutritional assessment and is reported to be significantly associated with the prognosis of colorectal cancer [50]. Additionally, the preoperative CONUT score was an independent prognostic factor for cancer specific and disease-free survival in obstructive colorectal cancer patients [22]. Results of this study suggest that presarcopenia is still considered to be an important factor in obstructive colorectal cancer, regardless of the patient's nutritional status or inflammatory condition.

In conclusion, a careful consideration and analysis of the patient's body composition status should be performed to overcome the large clinical burden in patients with obstructive colon cancer. In particular, pre-sarcopenia should be considered in the patients' risk assessments and stratification of oncologic prognosis. The pre-sarcopenia, diagnosed by L3SMI, may be beneficial as a convenient, objective, and noninvasive marker, to guide individualized treatment decisions and improve follow-up outcomes in patients with colorectal cancer.

\section{Abbreviations}

CT: Computed tomography; BMI: Body mass index; NLR: Neutrophil to lymphocyte ratio; DFS: Disease-free survival; OS: Overall survival; SMI: Skeletal muscle index; SEMS: Self-expandable metallic stent

\section{Acknowledgements}

We thank Soo Ji Park and other colleagues who helped with data collection in the colorectal division of the Surgery Department at The Catholic

University of Korea.

\section{Authors' contributions}

Lee CS and Oh ST designed the research; Lee CS, Won DD, Oh SN, Lee YS, Lee IK, Kim IH, and Choi MH performed the clinical research; Won DD and Oh SN analyzed data; Lee CS and Won DD wrote the paper. The authors read and approved the final manuscript.

\section{Funding}

There is no funding of this study.

\section{Availability of data and materials}

The study data is not available

\section{Ethics approval and consent to participate}

All procedures performed in this study were in accordance with the ethical standards of the Institutional Review Board at the College of Medicine. This study was approved by The Catholic University of Korea Seoul St. Mary's Hospital Research Ethics Board (IRB: KC19RESI0152) and with the 1964 Helsinki Declaration and its later amendments or comparable ethical standard. Informed consent from patients to be included in this study was omitted according to the policy of our IRB.

\section{Consent for publication}

Informed consent from patients to be included in this study was omitted according to the policy of our IRB.

\section{Competing interests}

The authors declare that they have no competing interests

\section{Author details}

'Department of Surgery, Seoul St. Mary's Hospital, College of Medicine, The Catholic University of Korea, Seoul, Republic of Korea. ${ }^{2}$ Graduate School of Medicine, The Catholic University of Korea, Seoul, Republic of Korea.

${ }^{3}$ Department of Radiology, Seoul St. Mary's Hospital, College of Medicine, The Catholic University of Korea, Seoul, Republic of Korea. ${ }^{4}$ Cancer Research Institute, College of Medicine, The Catholic University of Korea, Seoul, Republic of Korea. ${ }^{5}$ Division of Medical Oncology, Department of Internal Medicine, Seoul St. Mary's Hospital, College of Medicine, The Catholic University of Korea, Seoul, Republic of Korea. ${ }^{6}$ Department of Radiology, Eunpyeong St. Mary's Hospital, College of Medicine, The Catholic University of Korea, Seoul, Republic of Korea. ${ }^{7}$ Department of Surgery, Uijeongbu St. Mary's Hospital, College of Medicine, The Catholic University of Korea, 271, Cheonbo-Ro, Uijeongbu-si, Gyeonggi-do, 11765 Seoul, Republic of Korea.

Received: 6 June 2020 Accepted: 18 August 2020

Published online: 28 August 2020

References

1. Rosenberg $\mathbb{H}$. Sarcopenia: origins and clinical relevance. J Nutr. 1997;127: 990 s-1 s.

2. Fuggle N, Shaw S, Dennison E, Cooper C. Sarcopenia. Best Pract Res Clin Rheumatol. 2017:31:218-42.

3. Narici MV, Maffulli N. Sarcopenia: characteristics, mechanisms and functional significance. Br Med Bull. 2010;95:139-59.

4. Bozzetti F. Forcing the vicious circle: sarcopenia increases toxicity, decreases response to chemotherapy and worsens with chemotherapy. Ann Oncol. 2017:28:2107-18.

5. Batsis JA, Sahakyan KR, Rodriguez-Escudero JP, Bartels SJ, Lopez-Jimenez F. Normal weight obesity and functional outcomes in older adults. Eur J Intern Med. 2014;25:517-22. 
6. Baumgartner RN, Wayne SJ, Waters DL, Janssen I, Gallagher D, Morley JE. Sarcopenic obesity predicts instrumental activities of daily living disability in the elderly. Obes Res. 2004;12:1995-2004.

7. Batsis JA, Mackenzie TA, Barre LK, Lopez-Jimenez F, Bartels SJ. Sarcopenia, sarcopenic obesity and mortality in older adults: results from the National Health and Nutrition Examination Survey III. Eur J Clin Nutr. 2014:68:1001-7.

8. van Vugt $J \mathrm{~L}$, Braam $H J$, van Oudheusden TR, Vestering $A$, Bollen $T L$, Wiezer MJ, et al. Skeletal muscle depletion is associated with severe postoperative complications in patients undergoing cytoreductive surgery with hyperthermic intraperitoneal chemotherapy for peritoneal carcinomatosis of colorectal cancer. Ann Surg Oncol. 2015;22:3625-31.

9. Lieffers JR, Bathe OF, Fassbender K, Winget M, Baracos VE. Sarcopenia is associated with postoperative infection and delayed recovery from colorectal cancer resection surgery. Br J Cancer. 2012;107:931-6.

10. Mourtzakis M, Prado CM, Lieffers JR, Reiman T, McCargar $\amalg$, Baracos VE. A practical and precise approach to quantification of body composition in cancer patients using computed tomography images acquired during routine care. Appl Physiol Nutr Metab. 2008;33:997-1006.

11. Cruz-Jentoft AJ, Baeyens JP, Bauer JM, Boirie Y, Cederholm T, Landi F, et al. Sarcopenia: European consensus on definition and diagnosis: report of the European Working Group on Sarcopenia in Older People. Age Ageing. 2010; 39:412-23.

12. Mei KL, Batsis JA, Mills JB, Holubar SD. Sarcopenia and sarcopenic obesity: do they predict inferior oncologic outcomes after gastrointestinal cancer surgery? Perioper Med (Lond). 2016;5:30.

13. Simonsen $C$, de Heer P, Bjerre ED, Suetta C, Hojman P, Pedersen BK, et al. Sarcopenia and postoperative complication risk in gastrointestinal surgical oncology: a meta-analysis. Ann Surg. 2018;268:58-69.

14. Peng PD, van Vledder MG, Tsai S, de Jong MC, Makary M, Ng J, et al. Sarcopenia negatively impacts short-term outcomes in patients undergoing hepatic resection for colorectal liver metastasis. HPB (Oxford). 2011;13:439-46.

15. Choi MH, Oh SN, Lee IK, Oh ST, Won DD. Sarcopenia is negatively associated with long-term outcomes in locally advanced rectal cancer. J Cachexia Sarcopenia Muscle. 2018;9:53-9.

16. Cheynel N, Cortet M, Lepage C, Benoit L, Faivre J, Bouvier AM. Trends in frequency and management of obstructing colorectal cancers in a welldefined population. Dis Colon Rectum. 2007;50:1568-75.

17. Chen HS, Sheen-Chen SM. Obstruction and perforation in colorectal adenocarcinoma: an analysis of prognosis and current trends. Surgery. 2000; 127:370-6.

18. Chin CC, Wang JY, Changchien CR, Huang WS, Tang R. Carcinoma obstruction of the proximal colon cancer and long-term prognosis-obstruction is a predictor of worse outcome in TNM stage II tumor. Int Colorectal Dis. 2010;25:817-22.

19. McArdle CS, Hole DJ. Emergency presentation of colorectal cancer is associated with poor 5-year survival. Br J Surg. 2004;91:605-9.

20. Okuda Y, Shimura T, Yamada T, Hirata Y, Yamaguchi R, Sakamoto E, et al. Colorectal obstruction is a potential prognostic factor for stage II colorectal cancer. Int J Clin Oncol. 2018;23:1101-11.

21. Ignacio de Ulíbarri J, González-Madroño A, de Villar NG, González P, González B, Mancha A, et al. CONUT: a tool for controlling nutritional status. First validation in a hospital population. Nutr Hosp. 2005;20:38-45.

22. Sato R, Oikawa M, Kakita T, Okada T, Abe T, Yazawa T, et al. The Controlling Nutritional Status (CONUT) Score as a prognostic factor for obstructive colorectal cancer patients received stenting as a bridge to curative surgery. Surg Today. 2020.

23. Terzić J, Grivennikov S, Karin E, Karin M. Inflammation and colon cancer. Gastroenterology. 2010;138:2101-14.e5.

24. Mei Z, Liu Y, Liu C, Cui A, Liang Z, Wang G, et al. Tumour-infiltrating inflammation and prognosis in colorectal cancer: systematic review and meta-analysis. Br J Cancer. 2014;110:1595-605.

25. Medina Fernández FJ, Muñoz-Casares FC, Arjona-Sánchez A, Casado-Adam A, Gómez-Luque I, Garcilazo Arismendi DJ, et al. Postoperative time course and utility of inflammatory markers in patients with ovarian peritoneal carcinomatosis treated with neoadjuvant chemotherapy, cytoreductive surgery, and HIPEC. Ann Surg Oncol. 2015;22:1332-40.

26. Shibutani M, Maeda K, Nagahara H, Ohtani H, Iseki Y, Ikeya T, et al. The prognostic significance of a postoperative systemic inflammatory response in patients with colorectal cancer. World J Surg Oncol. 2015;13:194.

27. Kishi Y, Kopetz S, Chun YS, Palavecino M, Abdalla EK, Vauthey JN. Blood neutrophil-to-lymphocyte ratio predicts survival in patients with colorectal liver metastases treated with systemic chemotherapy. Ann Surg Oncol. 2009;16:614-22.

28. KızItan R, Yılmaz Ö, Aras A, Çelik S, Kotan Ç. Factors affecting mortality in emergency surgery in cases of complicated colorectal cancer. Med Glas (Zenica). 2016;13:62-7.

29. Alvarez JA, Baldonedo RF, Bear IG, Truán N, Pire G, Alvarez P. Presentation, treatment, and multivariate analysis of risk factors for obstructive and perforative colorectal carcinoma. Am J Surg. 2005;190:376-82.

30. Liska D, Stocchi L, Karagkounis G, Elagili F, Dietz DW, Kalady MF, et al. Incidence, patterns, and predictors of locoregional recurrence in colon cancer. Ann Surg Oncol. 2017;24:1093-9.

31. Mitsiopoulos N, Baumgartner RN, Heymsfield SB, Lyons W, Gallagher D, Ross R. Cadaver validation of skeletal muscle measurement by magnetic resonance imaging and computerized tomography. J Appl Physiol (1985). 1998:85:115-22.

32. Takagi K, Yagi T, Yoshida R, Shinoura S, Umeda Y, Nobuoka D, et al. Sarcopenia and American Society of Anesthesiologists Physical Status in the assessment of outcomes of hepatocellular carcinoma patients undergoing hepatectomy. Acta Med Okayama. 2016:70:363-70.

33. Heus C, Cakir H, Lak A, Doodeman HJ, Houdijk AP. Visceral obesity, muscle mass and outcome in rectal cancer surgery after neo-adjuvant chemoradiation. Int J Surg. 2016;29:159-64.

34. Saida Y, Sumiyama Y, Nagao J, Uramatsu M. Long-term prognosis of preoperative "bridge to surgery" expandable metallic stent insertion for obstructive colorectal cancer: comparison with emergency operation. Dis Colon Rectum. 2003;46:544-9.

35. Biolo G, Cederholm T, Muscaritoli M. Muscle contractile and metabolic dysfunction is a common feature of sarcopenia of aging and chronic diseases: from sarcopenic obesity to cachexia. Clin Nutr. 2014;33:737-48.

36. Jensen GL, Hsiao PY. Obesity in older adults: relationship to functional limitation. Curr Opin Clin Nutr Metab Care. 2010;13:46-51.

37. Rubio-Ruiz ME, Guarner-Lans V, Pérez-Torres I, Soto ME. Mechanisms underlying metabolic syndrome-related sarcopenia and possible therapeutic measures. Int J Mol Sci. 2019;20.

38. Hopkins JJ, Reif RL, Bigam DL, Baracos VE, Eurich DT, Sawyer MB. The impact of muscle and adipose tissue on long-term survival in patients with stage I to III colorectal cancer. Dis Colon Rectum. 2019;62:549-60.

39. Prado CM, Lieffers JR, McCargar $L$, Reiman $T$, Sawyer MB, Martin $L$, et al. Prevalence and clinical implications of sarcopenic obesity in patients with solid tumours of the respiratory and gastrointestinal tracts: a populationbased study. Lancet Oncol. 2008;9:629-35.

40. Morimoto Y, Maskarinec G, Conroy SM, Lim U, Shepherd J, Novotny R. Asian ethnicity is associated with a higher trunk/peripheral fat ratio in women and adolescent girls. J Epidemiol. 2012;22:130-5.

41. Malietzis G, Currie AC, Athanasiou T, Johns N, Anyamene N, Glynne-Jones R, et al. Influence of body composition profile on outcomes following colorectal cancer surgery. Br J Surg. 2016;103:572-80.

42. Kang BM, Kim HJ, Kye BH, Lee SC, Lee KY, Park SJ, et al. Multicenter, randomized single-port versus multiport laparoscopic surgery (SIMPLE) trial in colon cancer: an interim analysis. Surg Endosc. 2018;32:1540-9.

43. Moon HG, Ju YT, Jeong CY, Jung EJ, Lee YJ, Hong SC, et al. Visceral obesity may affect oncologic outcome in patients with colorectal cancer. Ann Surg Oncol. 2008:15:1918-22.

44. Clark W, Siegel EM, Chen YA, Zhao X, Parsons CM, Hernandez JM, et al. Quantitative measures of visceral adiposity and body mass index in predicting rectal cancer outcomes after neoadjuvant chemoradiation. J Am Coll Surg. 2013;216:1070-81.

45. Harimoto N, Shirabe K, Yamashita Yl, Ikegami T, Yoshizumi T, Soejima Y, et al. Sarcopenia as a predictor of prognosis in patients following hepatectomy for hepatocellular carcinoma. Br J Surg. 2013;100:1523-30.

46. Higashi T, Hayashi H, Taki K, Sakamoto K, Kuroki H, Nitta H, et al. Sarcopenia, but not visceral fat amount, is a risk factor of postoperative complications after major hepatectomy. Int J Clin Oncol. 2016;21:310-9.

47. Zhuang CL, Huang DD, Pang WY, Zhou CJ, Wang SL, Lou N, et al. Sarcopenia is an independent predictor of severe postoperative complications and longterm survival after radical gastrectomy for gastric cancer: analysis from a largescale cohort. Medicine (Baltimore). 2016;95:e3164.

48. Carvalho do Nascimento PR, Poitras S, Bilodeau M. How do we define and measure sarcopenia? Protocol for a systematic review. Syst Rev. 2018;7:51.

49. Fox $B$, Henwood $T$, Schaap $L$, Bruyère $O$, Reginster JY, Beaudart $C$, et al. Adherence to a standardized protocol for measuring grip strength and 
appropriate cut-off values in adults over 65 years with sarcopenia: a systematic review protocol. JBI Database System Rev Implement Rep. 2015;13:50-9.

50. Ahiko Y, Shida D, Horie T, Tanabe T, Takamizawa Y, Sakamoto R, et al. Controlling nutritional status (CONUT) score as a preoperative risk assessment index for older patients with colorectal cancer. BMC Cancer. 2019;19:946

\section{Publisher's Note}

Springer Nature remains neutral with regard to jurisdictional claims in published maps and institutional affiliations.

Ready to submit your research? Choose BMC and benefit from:

- fast, convenient online submission

- thorough peer review by experienced researchers in your field

- rapid publication on acceptance

- support for research data, including large and complex data types

- gold Open Access which fosters wider collaboration and increased citations

- maximum visibility for your research: over $100 \mathrm{M}$ website views per year

At $B M C$, research is always in progress.

Learn more biomedcentral.com/submissions 